\title{
Cutinase purification on poly(ethylene glycol)-hydroxypropyl starch aqueous two-phase systems
}

\author{
M.C. Almeida ${ }^{\mathrm{a}}$, A. Venâncio ${ }^{\mathrm{a}}$, J.A. Teixeira ${ }^{\mathrm{a}, *}$, M.R. Aires-Barros ${ }^{\mathrm{b}}$ \\ ${ }^{a}$ Departamento de Engenharia Biológica, Universidade do Minho, Campus de Gualtar, Largo do Paco, 4709 Braga Codex, Portugal \\ ${ }^{\mathrm{b}}$ Laboratório de Engenharia Bioquímica, Centro de Engenharia Biológica e Química, Instituto Superior Técnico, 1000 Lisbon, Portugal
}

\begin{abstract}
The partition behaviour of cutinase on poly(ethylene glycol) (PEG)-hydroxypropyl starch aqueous two-phase systems was characterized. The effect of molecular mass of PEG, the $\mathrm{pH}$ of the system and tie-line length on cutinase partition coefficient and cutinase yield to the top phase was investigated for systems prepared with a purified hydroxypropyl starch (Reppal PES 100) and a crude one (HPS). The effect of the presence of different salts, such as sodium chloride, sodium sulphate and ammonium sulphate, on cutinase partition was also studied. The results lead to the conclusion that aqueous two-phase systems composed of PEG and hydroxypropyl starch are not efficient in the purification of cutinase. In the majority of cases, the partition coefficients were very close to 1 , with $\mathrm{pH}$ being the factor which affects most cutinase partition. Partition coefficients were significantly improved when salts were added to the systems. For PEG 4000-Reppal PES 100 [at pH 4.0; $0.5 \mathrm{M}\left(\mathrm{NH}_{4}\right)_{2} \mathrm{SO}_{4}$ ], the partition coefficient for cutinase was 3.7, while a value of 12 was obtained for PEG 4000-HPS (at $\mathrm{pH} 4.0 ; 1 \mathrm{M} \mathrm{NaCl})$. An isoelectric point ( $\mathrm{p} I)$ of 7.8 was confirmed for cutinase by constructing a cross partition graphic from the results obtained in the experiments with different salts. (C) 1998 Elsevier Science B.V. All rights reserved.
\end{abstract}

Keywords: Aqueous two-phase systems; Enzymes; Cutinase; Poly(ethylene glycol)-hydroxypropyl starch

\section{Introduction}

Pharmaceutical, detergent and food industries show an increasing interest in the use of lipolytic enzymes. Fusarium solani pisi cutinase, cloned and expressed in Escherichia coli, has a great potential for industrial use due to its lipolytic activity, considerable thermostability and versatility [1]. Unlike lipases, cutinase can catalyse the hydrolysis of lipids in aqueous solutions, i.e., in the absence of a lipidwater interface [2].

After producing this enzyme by fermentation, $E$.

\footnotetext{
*Corresponding author.
}

coli cells are harvested and cutinase is removed from the periplasmic space by osmotic shock. Then, a sequence of purification steps must be followed, namely an acid precipitation and chromatographic methods, which make this process very expensive and time consuming. As an alternative to the first purification step, the fermentation broth could be subjected to a purification process based on aqueous two-phase systems (ATPSs).

ATPSs are useful for separations involving labile biological materials, mainly because of their high water content [3]. The extraction processes using ATPSs are easily applied as downstream procedures: no expensive specialised equipment is necessary, they can be scaled up and operated on a continuous basis [4]. Protein purification using ATPSs is in- 
fluenced by a number of parameters, such as the system $\mathrm{pH}$, the type and concentration of salts present in the system, the polymer molecular mass and concentration and protein properties (e.g., structure, hydrophobicity, molecular mass) [3]. Thus far, most of the laboratory work has been made with a system composed of fractionated dextran and poly(ethylene glycol) (PEG) [5]. The high cost of the fractionated dextran is a drawback for its application in large-scale processes. However, some other polysaccharides were tested, which could be successful in the replacement of fractionated dextran: starch derivatives [6,7], poly(vinyl alcohol) [8], maltodextrin [9], cellulose derivatives [10], poly(ethyleneimine) [11], agarose [12], guar gum [13] and locust bean gum [14]. As an alternative to polymer-polymer ATPS, PEG-salt systems have been used. Though inexpensive, the high ionic strength resulting from the high salt concentration can cause protein denaturation and the use of ligands is not possible.

Some work has already been done on cutinase extraction by ATPS, using PEG and potassium phosphate systems [15]. After the recombinant $E$. coli cells harvest, cells were disrupted and the homogenate was subjected to purification using an ATPS. As an outcome of this purification procedure, there was a set of results of cutinase extraction showing a high purification factor from cell debris and a high lipolytic activity yield for the top phase, at $\mathrm{pH} 4.8$ with a system composed of $25 \%$ PEG 1000-10\% sodium sulphate [15]. Hence, this method seems to be an interesting alternative to the procedures described by Lauwereys et al. [1]. However, no further work with ATPSs has been done. Thus, it is necessary to evaluate the applicability of ATPSs composed of PEG and a polymer in cutinase extraction. Crude starch derivatives are one of the most interesting alternatives.

In the present work, systems composed of PEG and a crude hydroxypropyl starch (HPS) are studied and compared with ATPSs formed by PEG and Reppal PES 100 (a purified hydroxypropyl starch), to evaluate their performance in the extraction of cutinase. These experiments were carried out using a purified cutinase and the characterization of the main factors that affect cutinase partition behaviour on the PEG-HPS ATPS was studied.

\section{Experimental}

\subsection{Chemicals}

PEG average molecular mass of $(7-9) \cdot 10^{3} \mathrm{Da}$ (PEG 8000) and average molecular mass of $1000 \mathrm{Da}$ (PEG 1000) were purchased from Sigma (St. Louis, MO, USA). PEG average molecular mass of $4 \cdot 10^{3}$ Da (PEG 4000) was purchased from Merck (Darmstadt, Germany). Reppal PES 100 (Rep) was obtained from Reppe (Vaxjo, Sweden). Crude hydroxypropyl starch (HPS) was a generous gift from Sarcol (Porto, Portugal). All other chemicals were analytical grade and water was distilled and passed through a mixed ion exchanger.

\subsection{Proteins}

Bovine serum albumin (BSA) was obtained from Sigma. Cutinase from Fusarium solani pisi was produced from the E. coli WK-6 recombinant strain (which was a kind gift of Corvas International, Gent, Belgium), purified and lyophilised at the Laboratório de Engenharia Bioquímica, Instituto Superior Técnico (UTL) according to Lauwereys et al. [1].

\subsection{Two-phase systems}

The systems were prepared from stock solutions of polymers in water. Concentrations of $70 \%, 60 \%$ and $50 \%(\mathrm{w} / \mathrm{w})$ were prepared, for PEG 1000, PEG 4000 and PEG 8000, respectively. A 30\% (w/w) stock solution of Reppal PES 100 was prepared by dissolving the powder in cold water.

The HPS stock solution was prepared by dissolving the powder in hot water. This solution was maintained under continuous agitation at $80^{\circ} \mathrm{C}$ for 30 min, and centrifuged (4000 $\mathrm{g}, 30 \mathrm{~min}, 4^{\circ} \mathrm{C}$ ), to eliminate insoluble materials. The correct HPS concentration was determined after hydrolysis with sulphuric acid, by measuring the reducing sugars using the method developed by Miller [16].

For system preparation, polymer solutions were weighed and mixed with water, buffer and $250 \mu$ of a $5 \mathrm{mg} / \mathrm{ml}$ cutinase solution. Systems containing salts were prepared by adding the correct amount of the salt stock solution to the previous mixture, for 
salt concentrations of less than $0.5 \mathrm{M}$, or by dissolving powdered salt directly into the systems, for salt concentrations of $0.5 \mathrm{M}$ or $1 \mathrm{M}$. The total mass of the systems was adjusted to $5.0 \mathrm{~g}$ by adding water. In all systems, the buffer concentration was $10 \mathrm{~m} M$ and the temperature for phase separation was $4^{\circ} \mathrm{C}$. Phase separation was achieved by centrifugation, 2-3 min at $4000 \mathrm{~g}$. Both phases were weighed and analysed in order to determine protein concentration and cutinase activity.

\subsection{Protein and cutinase assays}

Protein concentration on the top phase was determined according to Bradford [17], using BSA as standard.

Cutinase activity was measured according to Wincker [18]: 1 unit ( $1 \mathrm{U})$ of cutinase activity was defined as the amount of enzyme that catalyses the production of $1 \mathrm{nmol}$ of $p$-nitrophenol/ml in $1 \mathrm{~min}$ from $p$-nitrophenol palmitate at $24 \pm 1^{\circ} \mathrm{C}$. For the cutinase assays, the top phase sample was diluted 10-times in water. The protein concentration and cutinase activity/ml for the bottom phase were calculated from mass balance.

To describe cutinase partition, three parameters were determined: the protein partition coefficient, defined as the ratio between the protein top and bottom phase concentrations $\left(K_{\mathrm{p}}\right)$, the cutinase partition coefficient, defined as the ratio between the top and bottom phase cutinase activity/ml $\left(K_{\text {cut }}\right)$ and the cutinase yield for the top phase calculated by:

$$
Y(\%)=\frac{100}{1+\frac{V_{\text {bottom }}}{V_{\text {top }}} \times \frac{1}{K_{\text {cut }}}}
$$

\section{Results and discussion}

Several ATPSs formed by Reppal PES 100 or crude HPS with PEG were tested for their ability to extract cutinase. For each system, the following effects were studied: $\mathrm{pH}(4.0,6.0,8.0,9.0)$, PEG average molecular mass $(1000,4000,8000)$ and tie-line length.

The presence of different salts (sodium chloride, sodium sulphate and ammonium sulphate) on cutin- ase partition was also assayed for ATPSs formed by PEG 4000-Reppal PES 100 and PEG 4000-HPS at different $\mathrm{pH}$ values.

It is shown that systems formed by PEG and crude HPS may be considered as an interesting alternative for cutinase purification, if the correct salt is selected.

\subsection{PEG-Reppal PES 100 ATPSs}

Polymer compositions of tested systems are shown in Table 1. For these systems, the top phase volume varies between $55 \%$ and $65 \%$ of the total volume of the system. The effect of PEG average molecular mass, tie-line length and $\mathrm{pH}$ on the three parameters studied $\left[K_{\mathrm{p}}, K_{\text {cut }}\right.$ and $\left.Y(\%)\right]$ is shown in Fig. 1.

In most of the 36 cases tested situations, the cutinase partition coefficient and the protein partition coefficient (Fig. 1) range from 0.5 to 1 . Clearly, for an efficient cutinase extraction, these values should be greater than 1, which is not the case for the tested values of PEG average molecular mass, tie-line length and $\mathrm{pH}$. Concerning cutinase partition, the best result was obtained for the PEG 8000-Reppal system at $\mathrm{pH} 8.0$ and for the medium length tie-line. Values of the same order of magnitude, but slightly lower, were obtained for the PEG 4000-Reppal system at $\mathrm{pH} 8.0$ and 9.0 and PEG 8000-Reppal system at $\mathrm{pH}$ 9.0, for medium length tie-line. These results clearly demonstrate the reduced effect of system properties in cutinase partition.

Table 1

Compositions (w/w) of PEG-Reppal systems with PEG 1000, PEG 4000 and PEG 8000

\begin{tabular}{llll}
\hline System & Tie-line & PEG $(\%)$ & Reppal $(\%)$ \\
\hline PEG 1000 & Short & 12 & 13 \\
& Medium & 13 & 13 \\
& Long & 15 & 14 \\
PEG 4000 & Short & 5.5 & \\
& Medium & 7.5 & 13 \\
& Long & 10 & 13 \\
PEG 8000 & Short & 5.1 & 14 \\
& Medium & 7.0 & 13 \\
& Long & 9.2 & 13 \\
& & & 14 \\
\hline
\end{tabular}




\section{PEG8000}

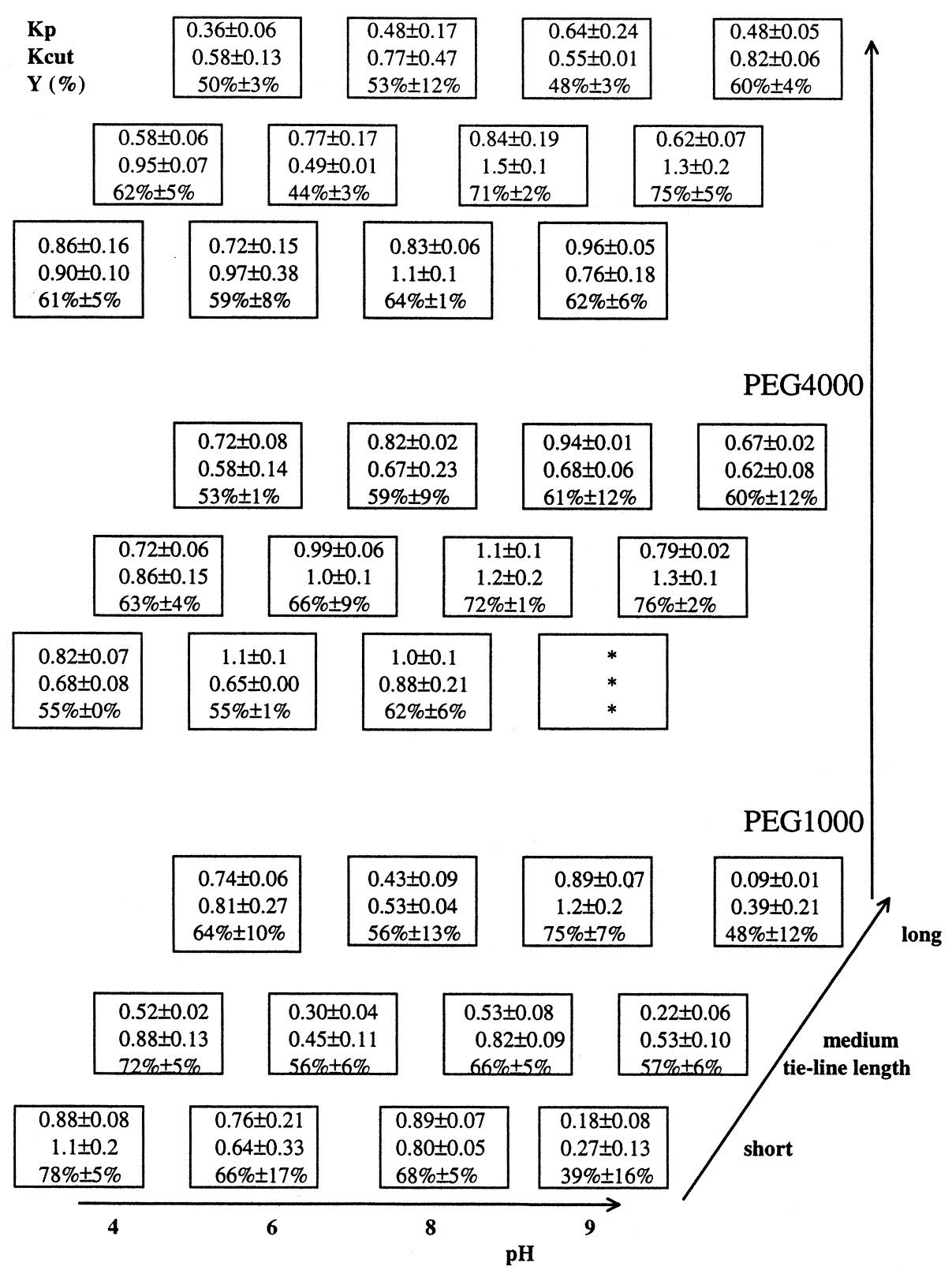

Fig. 1. Protein partition coefficient, cutinase partition coefficient and the percent yield for the top phase for systems composed of Reppal and PEG.

\subsection{1. $p H$ effect}

Although $K_{\mathrm{p}}, K_{\text {cut }}$ and $Y$ show little changes by changing the system $\mathrm{pH}$, a slight increase of these parameters can be detected as the $\mathrm{pH}$ is increased from 4.0 to 8.0. Nevertheless, at $\mathrm{pH} 9.0$ these values are, in general, lower than for $\mathrm{pH}$ 8.0. This may be explained on the basis of the hydrophobic properties of PEG [19], that can bind to the hydrophobic 
residues of cutinase. Forciniti et al. [20] reported that hydrophobic interactions are stronger at a $\mathrm{pH}$ closer to the isoelectric point $(\mathrm{p} I)$, which for cutinase is 7.8 (as shown in Fig. 7).

Lower values were detected for the system with PEG 1000 at $\mathrm{pH}$ 9.0. Cutinase denaturation and precipitation probably occurred. With PEG of higher molecular mass, the protein is stabilised by the PEG which minimises the denaturating effects [3].

\subsubsection{PEG molecular mass}

The results presented in Fig. 1 indicate that the system studied is slightly influenced by the PEG molecular mass. The partition coefficients and the top phase yield show no regular tendency and are similar in all cases. Due to an excluded volume effect, the general tendency expected would be an increase of the partition coefficients and top phase yield as the PEG molecular mass decreases. However, it is important to bear in mind that by changing the polymer molecular mass, one needs to alter the polymer concentration (e.g., for PEG 1000 a greater amount of the polymer is needed to form an ATPS than for PEG 8000), which influences the partition coefficients in the opposite way.

Conversely, as the PEG molecular chain increases, its hydrophobic character becomes stronger [19]. Therefore, and considering that the cutinase has apolar groups at its surface, an increase of the PEG molecular mass should increase the cutinase affinity for the top, PEG-rich, phase. This fact explains the extremely low values for $K_{\mathrm{p}}, K_{\text {cut }}$ and $Y(\%)$ generally obtained with the PEG 1000-Reppal ATPS (e.g., $K_{\mathrm{p}}=0.30 ; K_{\text {cut }}=0.45 ; Y=56 \%$, for the medium length tie-line at $\mathrm{pH} 6$, and $K_{\mathrm{p}}=0.09 ; K_{\text {cut }}=0.21$; $Y=48 \%$, for the longer length tie-line at $\mathrm{pH} 9.0$ ).

Since partition behaviour is much more sensitive to polymer molecular mass for proteins with molecular masses higher than $50 \mathrm{kDa}$ [21], the low molecular mass of cutinase ( $22 \mathrm{kDa}$ ) may be responsible for the slight changes observed.

\subsubsection{Tie-line length effect}

This effect is related to the influence of the distance from the critical point on the partitioning behaviour of cutinase in ATPSs. Near the critical point, the $K_{\mathrm{p}}$ and $K_{\text {cut }}$ should be close to 1 [8], which is confirmed by most of the results shown in Fig. 1.
For systems prepared with PEG 8000, a slight decrease in $K_{\mathrm{p}}, K_{\text {cut }}$ and $Y(\%)$ is observed as the tie-line length is increased. This increase is due to the increasing PEG concentration (Table 1), which causes a molecular exclusion of the cutinase from the top to the bottom phase. Results obtained by Sturesson et al. [22] with two different proteins show the same behaviour as the tie-line length is increased.

\subsection{PEG-HPS ATPSS}

Polymer compositions of the tested PEG-HPS systems are shown in Table 2. In Fig. 2, $K_{\mathrm{p}}, K_{\text {cut }}$ and $Y(\%)$ are presented for the different cases tested. The values for the protein partition coefficient vary from 0.12 (PEG 1000, pH 4.0 and medium tie-line length) to 1.6 (PEG 1000, pH 8.0 and short tie-line length), the $\mathrm{pH}$ seems to be the factor that mostly affects cutinase partition.

The cutinase partition coefficient values vary between 0.16 (PEG 1000, pH 6.0 and long tie-line length) and 1.3 (PEG 4000, pH 9.0 and short tie-line length). For most of the tested situations $K_{\mathrm{p}}$ and $K_{\text {cut }}$ remain between 0.5 and 1 , as already observed for the PEG-Reppal ATPS. For $Y$, although the order of magnitude is the same, a slight decrease is observed.

\subsection{1. $p H$ effect}

Fig. 2 shows a general increase of the protein partition for the top phase as $\mathrm{pH}$ approaches 8.0. This partition behaviour is similar to the one for the PEG-Reppal ATPS. Although there is not a clear

Table 2

Compositions (w/w) of PEG-HPS systems with PEG 1000, PEG 4000 and PEG 8000

\begin{tabular}{llcl}
\hline System & Tie-line & PEG $(\%)$ & HPS $(\%)$ \\
\hline PEG 1000 & Short & 9.1 & 10 \\
& Medium & 12 & 10 \\
& Long & 14 & 10 \\
PEG 4000 & Short & 5.7 & 10 \\
& Medium & 7.4 & 10 \\
& Long & 9.1 & 10 \\
PEG 8000 & Short & 5.1 & 10 \\
& Medium & 7.0 & 10 \\
& Long & 9.2 & 10 \\
\hline
\end{tabular}




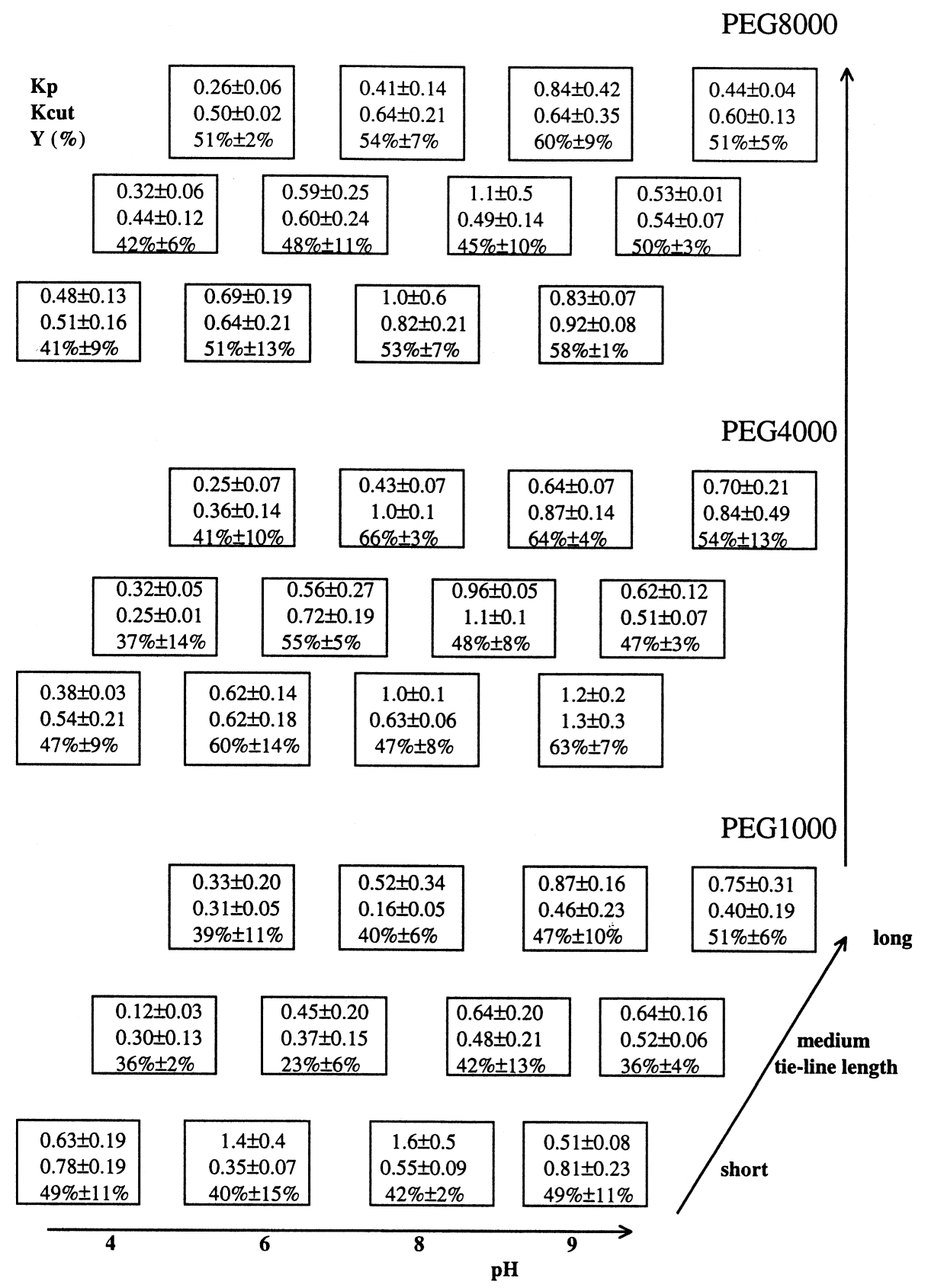

Fig. 2. Protein partition coefficient, cutinase partition coefficient and the cutinase yield on the top phase for systems composed of HPS and PEG.

trend for cutinase partition, lower values are obtained at $\mathrm{pH} 4.0$ and $6.0(<$ cutinase $\mathrm{pI})$. The different behaviour of these two parameters is either due to a loss of cutinase activity $\left(K_{\mathrm{p}}>K_{\text {cut }}\right)$ or to an increase of cutinase activity resulting from the stabilisation of cutinase through PEG $\left(K_{\text {cut }}>K_{\mathrm{p}}\right)$. 


\subsubsection{PEG molecular mass}

In all cases tested, there is not a regular trend for $K_{\mathrm{p}}, K_{\text {cut }}$ and $Y(\%)$ variation with PEG molecular mass. As already explained, for proteins with a low molecular mass $(<50 \mathrm{kDa})$, this effect has little influence on its partitioning behaviour. Furthermore, electrostatic interactions, which are stronger in PEGHPS than in PEG-Reppal due to the presence of impurities, can attenuate the effect of PEG molecular mass [20].

\subsubsection{Tie-line length effect}

The increase of the tie-line length (keeping constant the HPS concentration) leads, in most cases, to a decrease of $K_{\mathrm{p}}$. These results are in accordance with the data presented by Sturesson et al. [22]. For $K_{\text {cut }}$, this is not as clear. This variability of results (between 0.5 and 1) is probably due to the opposite effects of the excluded volume effect and the preferential affinity of cutinase for the hydrophobic phase (the lowest value for $K_{\text {cut }}$ was observed for the PEG 1000 systems - less hydrophobic, as already explained- for the longer length tie-line).

\subsection{Comparison between PEG-Reppal and PEG- HPS ATPSS}

Although it can be said that the results obtained for the PEG-HPS ATPS are very similar to the ones found for the PEG-Reppal system, some differences can be noted, especially when comparing the $\mathrm{pH}$ effect on the two systems (Figs. 3 and 4). Since the polymer used in both cases is a HPS, the differences must be related to: (i) the impurities present in crude HPS (e.g., ions that affect cutinase interactions with the ATPS); (ii) the different polymer concentration used to prepare the ATPS; (iii) the different values of polymer average molecular mass and polymer polydispersity.

\subsubsection{The effect of the presence of salts}

The presence of salts alters the cutinase-PEG hydrophobic interactions in a similar way as in hydrophobic interaction chromatography. In ATPSs, salts will be solvated by water molecules, hence facilitating hydrophobic interactions.

The results obtained for $K_{\text {cut }}$ in ATPSs in the presence of sodium chloride, ammonium sulphate

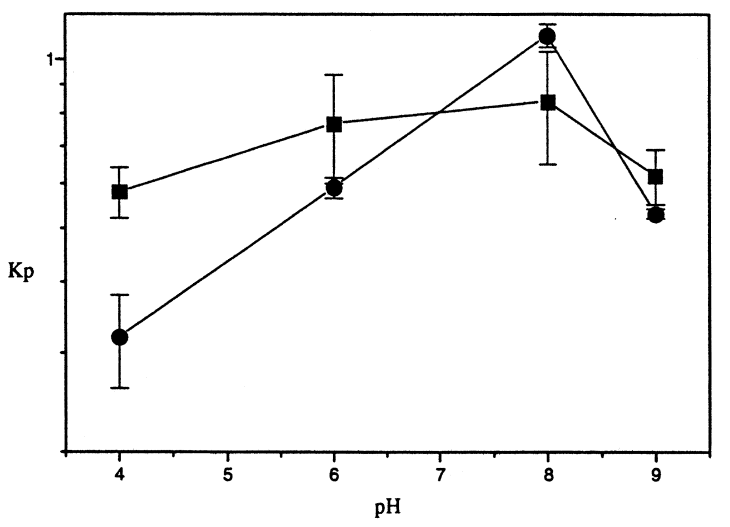

Fig. 3. pH effect on protein partition coefficient - ATPSs with PEG 8000 and Reppal (ם) or PEG 8000 and HPS (@) (medium tie-line).

and sodium sulphate are shown in Figs. 5 and 6. There are significant differences in $K_{\text {cut }}$ in the presence and in the absence of salts, mostly at extreme $\mathrm{pH}$ values (4.0 and 9.0). The cutinase affinity towards PEG depends on the type of salt present, which will change the electrical potential difference between the phases [3].

The results show that, in most cases (Figs. 5-7), $K_{\text {cut }}$ is higher in the presence of salts. As $\mathrm{pH}$ becomes more extreme ( $\mathrm{pH} 4.0$ and 9.0), the difference between $K_{\text {cut }}$ in systems with and without salts becomes significant. Around $\mathrm{pH} 8.0$ (close to cutinase $\mathrm{p} I), K_{\text {cut }}$ is less influenced by the type and

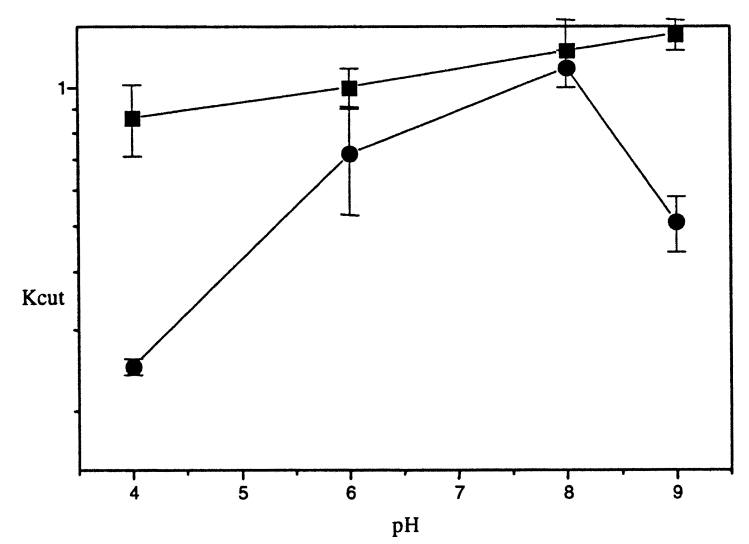

Fig. 4. $\mathrm{pH}$ effect on cutinase partition coefficient - ATPSs with PEG 8000 and Reppal (ם) or PEG 8000 and HPS (-) (medium tie-line). 


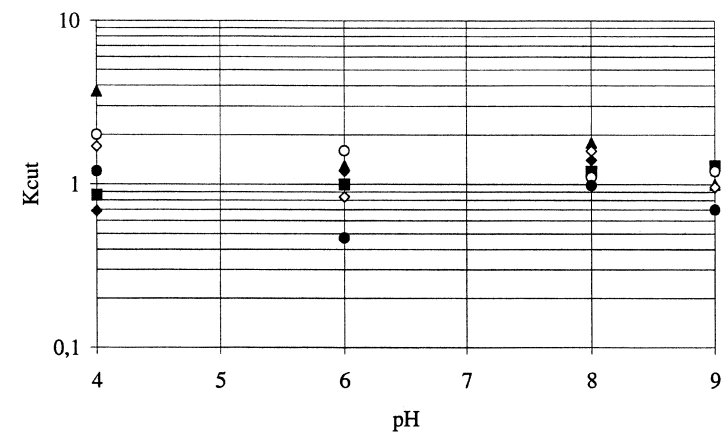

Fig. 5. Cutinase partition coefficient at different $\mathrm{pH}$ values in the presence of salts. ATPS 7.5\% PEG 4000-13\% Reppal; no salts (ם), $0.1 M$ sodium chloride $(\diamond), 0.05 M$ sodium sulphate $(\bigcirc)$, $0.5 M$ ammonium sulphate $(\boldsymbol{\Delta}), 1 M$ sodium chloride $(\diamond), 0.5 M$ sodium sulphate $(\mathbf{O})$.

concentration of the salts used. This agrees with previous results obtained in the presence of different salts at $\mathrm{pH}=\mathrm{p} I$ of the protein [3]. For PEG 4000Reppal systems, the higher partition coefficient for cutinase $(K=4)$ is obtained in the presence of ammonium sulphate and the lower in the presence of sodium chloride, these results being similar to the ones obtained by Albertsson for phycoerythrin [3].

According to Melander and Horváth [23], the hydrophobic interactions would become stronger by increasing the salt concentration. This is not revealed by the results in Figs. 5 and 6. A loss of cutinase activity might have occurred at a higher salt con-

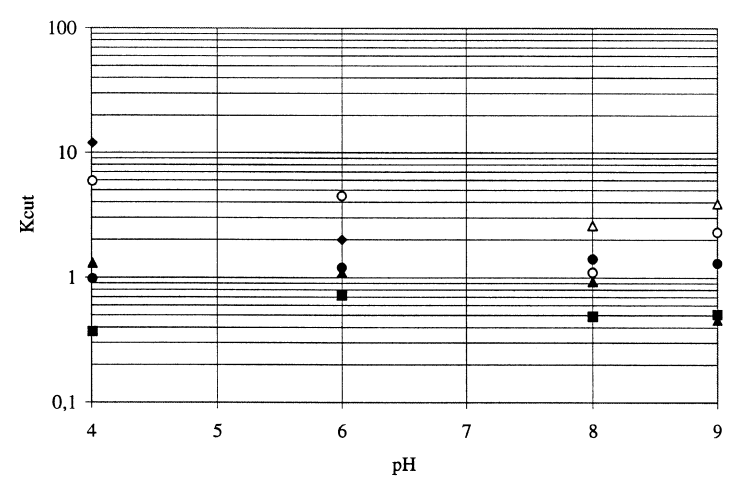

Fig. 6. Cutinase partition coefficient at different $\mathrm{pH}$ values in the presence of salts. ATPS 7.4\% PEG 4000-10\% HPS; no salts (ם), $0.05 M$ ammonium sulphate $(\triangle), 0.05 M$ sodium sulphate $(\bigcirc)$, $0.5 M$ ammonium sulphate $(\boldsymbol{\Delta}), 1 M$ sodium chloride $(\diamond), 0.5 M$ sodium sulphate $(\mathbf{O})$.

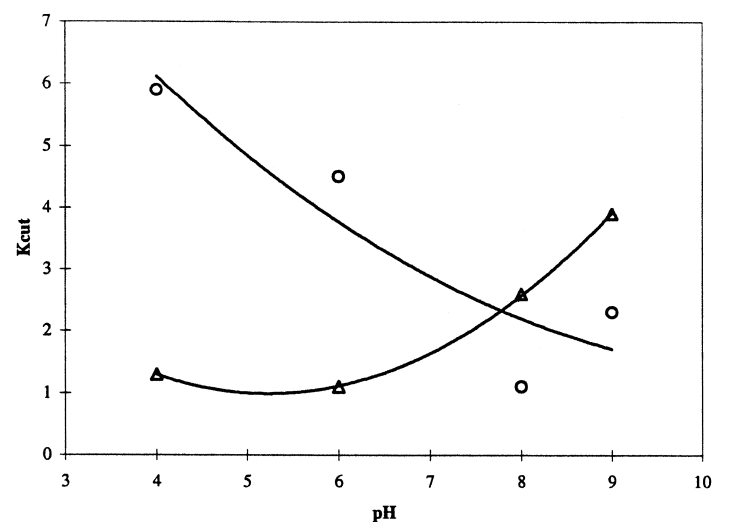

Fig. 7. Cross partition plot for cutinase in the presence of $0.05 \mathrm{M}$ ammonium sulphate and $0.05 M$ sodium sulphate. ATPS formed by $7.4 \%$ PEG $4000-10 \%$ HPS, $0.05 M$ ammonium sulphate $(\triangle)$, $0.05 M$ sodium sulphate $(\bigcirc)$.

centration, leading to lower values for the cutinase partition coefficient.

The cutinase partitioning behaviour in systems formed by PEG 4000-HPS is different from the one in the PEG 4000-Reppal ATPS. The maximum $K_{\text {cut }}$ $\left(K_{\text {cut }}=12\right)$ is at $\mathrm{pH} 4.0$, in the presence of $1 \mathrm{M} \mathrm{NaCl}$, although, in general, for this range of $\mathrm{pH}$ values, higher partition coefficients for cutinase are obtained in the presence of $0.05 \mathrm{M}$ sodium sulphate. Moreover, $K_{\text {cut }}$ values obtained in these systems present larger variations for the same $\mathrm{pH}$, than the ones obtained in the corresponding PEG-Reppal systems.

The differences between the PEG-Reppal and PEG-HPS ATPSs must be related to the impurities included in systems with the crude HPS. As already explained, they will change the ionic strength and consequently the electrical potential difference between the phases in the system, and thus the interaction of cutinase with the two phases of the ATPS.

\subsubsection{Cross partition results}

Protein $\mathrm{p} I$ and the $\mathrm{pH}$ of the solution are crucial factors in protein partitioning behaviour in ATPSs. Changes in the electrostatic strength in the system, leading to a higher variation of partition coefficients for a given $\mathrm{pH}$, can be obtained by adding salts.

A simple and easy way to determine protein $\mathrm{p} I$ is to perform cross partition experiments. The results 
obtained for cutinase partition in $7.4 \%$ PEG-10\% HPS ATPS at different $\mathrm{pH}$ values can be seen in Fig. 7. Two salts at the same concentration were tested and two lines were obtained by fitting the values of $K_{\text {cut }}$ to a second degree polynomial (correlation values of 0.9998 and 0.92 for the regression of values obtained with $\mathrm{Na}^{+}$and $\mathrm{NH}_{4}^{+}$, respectively).

These data show that the cutinase $\mathrm{pH}$ - determined from the intersection point of the lines - is 7.8 , which is in agreement with the value reported by Portela [24]. This Figure also illustrates the different way salts affect cutinase partition behaviour.

\section{Conclusions}

An efficient and inexpensive cutinase purification can be achieved in PEG-starch derivatives ATPSs, if the correct salt is selected. It is shown that salts have a strong contribution to cutinase partition behaviour.

For PEG-HPS ATPSs, in the absence of salts, the purification of cutinase is not efficient - the partition coefficients (in most of the experiments close to or below 1) and the yield for the top phase is low. Cutinase partition behaviour seems to be little influenced by changing PEG average molecular mass (from 1000 to 8000) and tie-line length, unlike what happens in systems formed by PEG-salts $[25,26]$.

There is a general increase of the partition coefficient with the system $\mathrm{pH}$, especially in ATPSs with HPS, whose impurities change the electrostatic interactions in the system. At $\mathrm{pH} 8.0, K_{\mathrm{p}}$ and $K_{\text {cut }}$ are generally higher than at the other $\mathrm{pH}$ values tested. This shows that a stronger cutinase attraction to the top, PEG-rich and more hydrophobic phase, occurs at a $\mathrm{pH}$ near its $\mathrm{p} I(7.8)$.

The presence of salts in the system changed remarkably the cutinase partitioning behaviour. The highest values for the cutinase partition coefficient occurred at $\mathrm{pH} 4.0$, with $0.5 \mathrm{M}$ ammonium sulphate in systems with Reppal and $1 M$ sodium chloride and $0.05 M$ sodium sulphate in systems with HPS.

At $\mathrm{pH} 8.0, K_{\text {cut }}$ shows little alteration with the different salt types and concentrations, because at this $\mathrm{pH}$ cutinase is near its $\mathrm{p} I$. Hence, it is less influenced by electrostatic changes produced by the salts.

\section{References}

[1] M. Lauwereys, P. De Geus, J. De Meutter, P. Stanssens and G. Matthyssens, in L. Alberghina, R.D. Schmid and R. Verger (Editors), Lipases: Structure, Mechanism and Genetic Engineering, Marcel Dekker, New York, 1990, Ch. 10, pp. 243-251.

[2] C. Martinez, P. De Geus, M. Lauwereys, M. Matthyssens, C. Cambillau, Nature 356 (1992) 615-618.

[3] P.-Å. Albertsson, Partition of Cell Particles and Macromolecules, Wiley, New York, 3rd ed., 1986.

[4] M.-R. Kula, K.H. Kroner, H. Hustedt, Adv. Biochem. Eng. 24 (1982) 74-118.

[5] P.-Å. Albertsson, G. Johansson and F. Tjerneld, in J.A. Asenjo (Editor), Separation Processes in Biotechnology, Marcel Dekker, New York Basel, 1990, Ch. 10, pp. 287-327.

[6] A. Venâncio, J.A. Teixeira, M. Mota, Biotechnol. Prog. 9 (1993) 635-638.

[7] F. Tjerneld, S. Berner, A. Cajarville, G. Johansson, Enzyme Microb. Technol. 8 (1986) 417-423.

[8] F. Tjerneld, in D. Fisher and I.A. Sutherland (Editors), Separation Using Aqueous Two-Phase Systems, Plenum Press, New York, 1989, Ch. 10, pp. 429-446.

[9] D.C. Szlag, K.A. Giuliano, Biotechnol. Techniques 2 (1988) 277-282.

[10] D.R. Skuse, R. Norris-Jones, M. Yalpani, D.E. Brooks, Enzyme Microb. Technol. 14 (1992) 785-790.

[11] U. Dissing, B. Mattiasson, Biotechnol. Appl. Biochem. 17 (1993) $15-21$

[12] A.S. Medin, J.-C. Janson, Carbohydr. Polymers 22 (1993) 127-136.

[13] A. Venâncio, C. Almeida, L. Domingues, J.A. Teixeira, Bioseparation 5 (1995) 253-258.

[14] A. Venâncio, C. Almeida, J.A. Teixeira, J. Chromatogr. B 680 (1996) 131-136.

[15] M.J. Sebastião, J.M.S. Cabral, M.R. Aires-Barros, Enzyme Microb. Technol. 18 (1996) 251-254.

[16] G.L. Miller, Anal. Chem. 31 (1959) 426-428.

[17] M.M. Bradford, Anal. Biochem. 72 (1976) 248-251.

[18] U.K. Wincker, J. Bacteriol. 138 (1979) 663-670.

[19] J. Huddleston, A. Veide, K. Kolher, J. Flanagen, S.-F. Enfors, A. Lyddiatt, Tibtech 9 (1991) 381-388.

[20] D. Forciniti, C.K. Hall, M.-R. Kula, Biotechnol. Bioeng. 26 (1992) 986-994.

[21] F. Tjerneld, in J.M. Harris (Editor), Poly(ethyleneglycol) Chemistry: Biotechnical and Biomedical Applications, Plenum Press, New York, 1992, pp. 85-102.

[22] S. Sturesson, F. Tjerneld, G. Johansson, Appl. Biochem. Biotechnol. 26 (1990) 281-295.

[23] W. Melander, C. Horváth, Arch. Biochem. Biophys. 183 (1977) 200-215.

[24] M.J.A.G. Portela, Síntese de Ésteres por Biocatálise Extractiva, MSc. Thesis (1996).

[25] M.J. Sebastião, J.M.S. Cabral, M.R. Aires-Barros, Biotechnol. Technol. 7 (1993) 631-634.

[26] M.J. Sebastião, J.M.S. Cabral, M.R. Aires-Barros, J. Chromatogr. A 668 (1994) 139-144. 